\section{On a problem of W. L. Ayres.}

By

\author{
Gordon T. Whyburn (Austin, Texas, U. S. A.).
}

In the paper Concerning continuous curves of certain types, (this: volume) W. L. Ayres raises the question as to whether or not every connected subset of a continuous curve $M$ having the property that for any positive number $\varepsilon, M$ contains not more than a finite number of simple closed curves of diameter greater than $\varepsilon$, is arewise connected. It is the purpose of the present paper to show that the answer to this question is affirmative.

In establishing the theorem below use will be made of the decomposition of a continuous curve into its cyclic elements, an extensive theory of which has been given by the author in a number of recent articles ${ }^{1}$ ). In particular the properties of the maximal cyclic curves of a continuous curve will be used.

Definitions. A conlinuous curve $M$ is suid to be cyclicly connected provided that every two points of $M$ lie together on some simple closed curve which is a subset of $M$. The continuous curve $C$ is-said to be a maximal cyclic curve of a continuous curve $M$ provided that $C$ is cyclicly connected and is a subset of $M$ but is not a proper subset of any cyclicly connected continuous curve which is also a subset of $M$. These definitions will be found in my papers just cited.

1) G. T. Whyburn, Cyclicly connected continuous curves. Proceedings of the National Academy of Sciences, vol. 13 (1927), pp. 31-38; Some properties of continuous curces, Bulletin of the American Mathematical Society, vol, 33 (1927), pp. 305-308; Concerning the structure of a continuous curve, presented to the American Mathematical Society December 31, 1926, offered to the American Journal of Mathematics, but not yet published.
In my paper Concerning the structure of a continuous curve I proved the following two propositions which are essential in the proof given below for Theorem [.

Theorem A. If $H$ is any connected subset of a continuous curve $M$ and $C$ is any maximal cyclic curve of $M$, then $H . C$ is either vacuous or connected.

Theorem B. In order that every connected subset of a continuous curve $M$ should be arcwise connected, it is necessary and sufficient that every connected subset of each maximal cyclic curve of $M$ should be arcwise connected.

The proof that the condition of Theorem $B$ is sufficient may be surnmarized as follows. Let $H$ be any connected subset of a continuons curre $M$ which satisfies the condition, and let $A$ and $B$ be any two points of $H$. Let $t$ be any arc in $M$ from $A$ to $B$ and $K$ the set of all those points of $M$ which separate ${ }^{1} A$ and $B$ in $M$. By a theorem of the anthor's ${ }^{2}$ ), the set of points $K+A+B$ is closed.

Clearly $K+A+B$ is a subset both of $t$ and of $H$. Hence $t-(K+A+B)$ is the sum of a countable number of maximal segments $S_{1}, S_{2}, S_{3}, \ldots$ For each positive integer $i, M$ contains ${ }^{3}$ ) a maximal cyclic curve $C_{i}$ containing $S_{i}$. Let $A_{i}$ and $B_{i}$ denote the endpoints of $S_{i}$. Now by Theorem $A$, for each $i$, the set of points $H . C_{i}$ is connected; and by hypothesis it follows that for each $i, H . C_{t}$ is arcwise connected. Then since, for each $i, H . C_{i}$ contains $A_{i}$ and $B_{i}$, therefore $H C_{t}$ contuins an arc $t_{i}$ from $A_{i}$ to $B_{i}$. Then the set of points

$$
T=K+A+B+\sum_{i=1,2,3 \ldots} t_{i}
$$

is a subset of $B$ and, using the properties of the maximal cyclic curves of a continuous carre established in my paper Cyclicty connected continuous curves (loc. cit), it is not difficult to show that $T$ is a simple continuous arc from $A$ to $B$. Hence $H$ is arcwise connected.

Theorem 1. If for every positive number $\varepsilon$, the continuous curve $M$ contains not snore than a finite number of simple closed curves of diameter greater than $\varepsilon$, then every connected subset of $M$ is arcwise connected.

1) The point $X$ of $M$ is said to separate the two points $A$ and $B$ in $M$ provided that $M-X$ is the sum of two mutually separated point sets containing $A$ and $B$ respectively.

2) Some properties of continuous curves, loc. cit., Theorem 1.

2) G. T. Whyburn, loc. cit., Theorem 2. 
Proof. Let $C$ be any maximal cyclic curve of $M$, and $A$ and $B$ any two points of $C$. Since $C$ is cyclicly connected, therefore it contains a simple closed curve $J$ containing both $A$ and $B$. The set of points $C-J$ consists of not more than a finite number of maximal connected subsets. For suppose, on the contrary, that there are infinitely many maximal counected subsets $R_{1}, R_{2}, R_{8}, \ldots$ of $C-J$. Then $J$ contains a point $P$ which is a limit point of the set of points $P_{1}+P_{4}+P_{3}+\ldots$, where for each integer $i>0$, $P_{i}$ is a point belonging to $R_{i}$. Let $X$ be a point of $J-P$ and let $2 d$ be the distance from $X$ to $P$. Then since, by a theorem of W. L. Ayres ${ }^{1}$ ), there are not more than a finite number of the sets $R_{i}$ of diameter greater than any preassigned positive number, it readily follows that there exists an infinite sequence of positive integers $n_{1}, n_{2}, n_{3}, \ldots$, such that for each $i$, every point of the set $R_{n_{i}}$ is at a distance less than $d$ from $P$. By a theorem of the author's ${ }^{2}$ ). for each $i, J$ contains at least two limit points of $R_{n_{i}}$. It easily follows with the aid of a theorem of R. L Moore's ') that for each $i, \bar{R}_{\mathbf{m}_{i}}$ contains an arc $t_{i}$ whose endpoints $A_{r}$ and $B_{i}$ belong to $J$, but such that $t_{i}-\left(A_{i}+B_{i}\right)$ belongs to $R_{n_{i}}$. Then for every positive integer $i$, the simple closed curve $J_{i}$ formed by the are $t_{i}$ plus the are $A_{i} X B_{i}$ of $J$ belongs to $C$ and is of diameter greater than $d$. But this is impossible since no two of the curves $\left[J_{i}\right]$ are identical and by hypothesis $M$ cannot contain more than a finite number of simple closed curves of diameter greater than $d$. Thus it follows that $C-J$ consists of not more than a finite number of maximal connected subsets. If there are any such sels, let them be ordered $R_{1}, R_{2}, R_{3}, \ldots, R_{n_{1}}$.

Let $i$ be any positive integer $\leqslant n_{1}$. Then $J$ contains only a finite number of limit points of $R_{i}$. For suppose it contains infinitely many such points. Then if $A X B$ and $A Y B$ denote the two ares of $J$ from $A$ to $B$, one of them, say $A X B$, must contain an infinite set $D$ of limit points of $R_{i}$. Let $E$ be a point of $D$. Then

1) W. L. A yres, Concerning continuous curves and correspondences, Annals of Mathematics, vol. 28 (1927), pp. 396-418, Theorem 1.

3) Cyclicly connected continwous curves, loc. cit., Theorem 9.

3) R. L. Moore, Concerning continuous curves in the plane, Mathematische. Zeitschrift, vol. 15 (1922), pp, 254-260, Theorem 1. with the aid of an accessibility theorem of R. L. Wilder's') is readily seen that $\bar{R}_{i}$ contains an infinite sequence of ares $\left[L^{\prime} X_{n} F_{n}^{\prime}\right]$, $n=1,2,3, \ldots$, no two of which are identical and where for every positive integer $n$, the point $F_{n}^{\prime}$ belongs to $\left.I\right)$ but $E X_{n} F_{n}-\left(E+F_{n}^{\prime}\right)$ belongs to $R_{i}$. Then for each positive integer $n$, the simple closed curve $J_{n}$ formed by the arc $E A Y B H_{n}^{\prime}$ of $J$ plus the are $k i X_{n} F_{n}$ belongs to $C$ and is of diameter $\geq$ the distance from $A$ to $B$. But this is impossible, since no two of the curves $\left[J_{n}\right]$ are identical and by hypothesis $M$ contains not more than a fite number of simple closed curves of diameter greater than any preassigned positive number. Thus the supposition that $J$ contains infinitely many limit points of $R_{i}$ leads to a coutradiction.

Now since there are just a finite number of the sets $\left[R_{i}\right]$, and since $J$ contains just a finite number of limit points of each of these sets, it follows that there exists an arc $U O I^{r}$ which is a subset of $J$ and which contains no limit point of $R_{1}+R_{2}+R_{3}+\ldots+R_{n_{1}}$.

For each positive integer $i \leq n_{1}$, it was seen above that $J$ contains at least two limit points of $K_{i}$. Then with the aid of R. L. Mo ore's theorem cited above it readily follows that for each $i \leq n_{1}$, $\bar{R}_{i}$ contains an are $t_{1 i}$ whose endpoints $A_{1 i}$ and $B_{1 i}$ are on $J$ but such that $t_{1 i}-\left(A_{1 i}+B_{1 i}\right)$ belongs to $K_{i}$. Let $K_{1}$ denote the set of points $J+\sum_{i=1}^{n_{1}} t_{1}$. Then just as in the case of $C-J$, it follows that $C-K_{1}$ is the sum of at most a finite number of maximal connected subsets $S_{1}, S_{2} ; S_{3}, \ldots, S_{n_{2}} ;$ and just as above it is shown that for every $i \leq n_{2}, \overline{S_{i}}$ contains an are $t_{2 i}$ whóse endpoints $A_{21}$ and $B_{2 i}$ are on $K_{1}$ but such that $t_{2 i}-\left(A_{2 i}+B_{2 i}\right)$ belongs to $S_{i}$. Let $K_{2}$ denote the set of points $K_{1}+\sum_{i=1}^{n_{2}} t_{2 i}$. Then $M-K_{2}$ is the sum of at most a finite number of maximal connected subsets $T_{1}, T_{2}, T_{3}, \ldots, T_{u_{3}}$. And each set $T_{i}$ contains an are $t_{3 i}$ whose endpoints $A_{3 i}$ and $B_{3 i}$ belong to $K_{2}$ but such that $t_{3 i}-\left(A_{3 i}+B_{3 i}\right)$ belongs to $T_{i}$. Let this process be continued. This process must terminate after a finite number of steps. For suppose it does not. Then $\sum_{n=1}^{\infty} \sum_{i=1}^{n_{n}} t_{n i}$ contains infinitely many different ares $\left[t_{n i]}\right]$. But each are $t_{n i}$ plus the set $K_{n-1}$

1) R. L. Wilder, Concerniny continuous" curves, this journal, vol. 7, (1925), pp. 3\$0-377, Theorem 1 
(where $K_{0}=J$ ) contains a simple closed curve $J_{n i}$ containing buth $t_{x i}$ and the arc UOV. And if $t_{l l,}$ and $t_{x y}$ are distinct arcs of the collection $\left[t_{n i}\right], a<x$, then since $J_{a b}$ is a subset of $K_{n-1}+t_{n b}$ and $J_{x y}$ is not a subset of this set of points (since $a<x$ ), therefore $J_{a b}$ and $J_{x y}$ are different simple closed curves. But each of simple closed curves $J_{n i}$ belongs to $C$ and contains UOV. This is contrary to the hypothesis that $M$ contains at most a finite number of simple closed curves of diameter greater than any preassigned positive number. Hence it follows that the above process must terminate after a finite number of steps, i. e., there exists a positive integer $k$ such that

$$
J+\sum_{n=1}^{k} \sum_{i=1}^{n_{n}} t_{n i}=C
$$

Let $K$ denote the set of points $A+B+\sum_{n=1}^{k} \sum_{i=1}^{n_{n}}\left(A_{n i}+B_{n i}\right)$.

Then $C-K$ consists of a finite number of its maximal connected subsets $s_{1}, s_{2}, s_{3}, \ldots, s_{n}$. It is easy to see that each of these sets $s_{i}$ is a segment of an arc $t_{i}$ whose endpoints belong to $K$, i. e., $t_{i}$ is the segment $s_{i}$ plus its two endpoints. Now if $N$ is any simple closed curve belonging to $C$, then for each positive integer $i<n$, $N$ either contains all of the segment $s_{i}$ or else it contains no point whatever of $s_{i}$. Hence every simple closed curve which $C$ contains is the sum of a certain number (two or more) of the arcs $t_{1}, t_{2}, t_{3}, \ldots, t_{n}$; and since there are only a finite number of these arcs, it follows that $C$ can contain only a finite number of simple closed curves. Then by a theorem due to W. L. Ayres ', it follows that every connected subset of $C$ is arcwise connected.

Now since, as I have just shown, every connected subset of each maximal cyclic curve of $M$ is arcwise connected, it follows by Theorem $B$ that every connected subset of $M$ is arcwise connected. This completes the proof of Theorem 1.

The following propositions either follow directly from the proof of Theorem 1, or else they follow by similar inethods and from the results of the paper of Ayres, using the decomposition of a continuous curve into its cyclic elements and the properties of

1) Concerning continuous curves of certain types, loc. cit., Theorem 81 have just shown that $C$ is "simply cyclic" in the sense of $A$ yres, $i$ e, that it contains only a finite number of simple closed curres those elements previously established by the author. For definitions

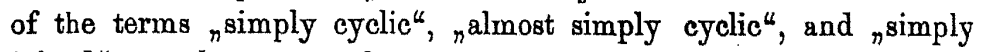
joined" see the paper of Ayres.

a) Every maximal cyclic curve of an almost simply cyclic continuous curve is simply cyclic.

b) Every maximal cyclic curve of an almost simply cyclic continuous curve is simply joined.

c) For cyclicly connected continuous curves, the properties nsimply cyclic", ${ }_{n}$ almost simply cyclic", and "simply joined" are equivalent.

d) If the cyclicly connected continuous curve $M$ is simply cyclic, and $K$ is any closed and connected subset of $M$, then $M-K$ is the sum of a finite. number of its maximal connected subsets each of which has only a finite number of limit points in $K$. Furthermore $M$ is the sum of a finite number of simple continuous arcs no two of which have an interior point of either in common.

The University of Texas, August 4, 1927. 